\title{
ImmunoCyt/uCyt $+{ }^{\mathrm{TM}}$ improves the sensitivity of urine cytology in patients followed for urothelial carcinoma
}

\author{
Bernard Têtu ${ }^{1}$, Rabi Tiguert ${ }^{2}$, François Harel $^{3}$ and Yves Fradet ${ }^{2}$ \\ ${ }^{1}$ Department of Pathology; ${ }^{2}$ Department of Urology and ${ }^{3}$ Department of Epidemiology, Centre Hospitalier \\ Universitaire de Québec, L’Hôtel-Dieu de Québec, Laval University, Québec, Canada
}

\begin{abstract}
ImmunoCyt/uCyt ${ }^{\mathrm{TM}}$ is a fluorescent test combining three monoclonal antibodies. In this study, it has been tested as a complement to cytology in the detection of urothelial carcinoma in urine. It has been performed simultaneously with standard cytology and cystoscopy on 870 urine analyses from one hospital. In 136 cases, one or more bladder tumors were found. Overall sensitivity of cytology, ImmunoCyt/uCyt ${ }^{\mathrm{TM}}$ and combined analyses reached 29,74 and $84 \%$, respectively, and overall specificity was 98 , 62 and $61 \%$. The negative predictive value of cytology, ImmunoCyt/uCyt ${ }^{\mathrm{TM}}$ and both analyses was 88,93 and $95 \%$, respectively, and the positive predictive value was 70,26 and $29 \%$. The sensitivity of cytology for low malignant potential neoplasms, low- and high-grade papillary carcinomas was 6,18 and $53 \%$, while it reached 71,79 and $93 \%$ when combined with ImmunoCyt/uCyt ${ }^{\mathrm{TM}}$. The sensitivity of cytology for stages Ta, T1, T2 and over and Tis tumors was12, 67, 47 and $50 \%$, while it reached $78,83,79$ and $100 \%$ when combined with ImmunoCyt/uCyt ${ }^{\mathrm{TM}}$. In the absence of tumor on cystoscopy but with positive ImmunoCyt/uCyt ${ }^{\mathrm{TM}}$, $18 \%$ of patients developed a tumor, 2-6 months later. Of the 109 cases diagnosed as suspicious for malignancy by cytology, a tumor was present in 30 cases and ImmunoCyt/uCyt ${ }^{\mathrm{TM}}$ was positive in $22(73 \%)$ of them. In conclusion, ImmunoCyt/uCyt ${ }^{\mathrm{TM}}$ may be used to postpone cystoscopies in patients followed for bladder cancer and may help to save cytologist and pathologist screening time.
\end{abstract}

Modern Pathology (2005) 18, 83-89, advance online publication, 24 September 2004; doi:10.1038/modpathol.3800262

Keywords: urothelial carcinoma; cytology; ImmunoCyt/uCyt ${ }^{\mathrm{TM}}$; immunofluorescence; urinary bladder; recurrence

Superficial bladder cancers are defined as tumors limited to the mucosa (Tis, Ta) or invading into the lamina propria (T1), without muscle invasion. ${ }^{1}$ They represent more than $80 \%$ of urothelial carcinomas and more than $50 \%$ will recur. ${ }^{1-3}$ Patients with bladder cancer are followed with regular cytologies and cystoscopies. Cytology is very specific but this test is limited by its low sensitivity ranging from 16 to $60 \%{ }^{4}$ Cytology is most useful at detecting highgrade (HG) cancer, whereas its sensitivity for lowgrade (LG) urothelial tumors is low and merely reaches $17 \% .{ }^{4}$ Furthermore, criteria used in urinary cytology to detect tumor cells are largely subjective and the ability to detect cancer cells is dependent on the experience of cytologists or pathologists. For instance, atypical cells and papillary aggregates may be found in either reactive or neoplastic conditions. ${ }^{5}$

Correspondence: Dr B Têtu, MD, Department of Pathology, Centre Hospitalier Universitaire de Québec, L'Hôtel-Dieu de Québec, 11, Côte du Palais, Québec, Canada G1R 2J6.

E-mail: bernard.tetu@chuq.qc.ca

Received 18 March 2004; revised and accepted 15 July 2004; published online 24 September 2004
Furthermore, LG urothelial carcinomas do not always exfoliate in aggregates and the cell characteristics are often so subtle that they might not be recognizable even by an experienced cytologist. ${ }^{5}$

Standard practice in the follow-up of patients with bladder cancer requires cystoscopies at regular intervals, but this technique is invasive, costly and causes discomfort to the patient. It is thus estimated that by improving the sensitivity of cytology, fewer follow-up cystoscopies would be needed. Furthermore, improved sensitivity would help cytologists and pathologists to save time currently spent at screening urinary smears, especially in cases with mild atypias and papillary aggregates of unknown significance. Moreover, one of the main interests of combining urinary cytology with cystoscopy is to identify nonvisible HG carcinoma in situ often missed by the cystoscopist and interpreted as suspicious by the cytologist.

ImmunoCyt/uCyt $^{\mathrm{TM}}$ (DiagnoCure Inc., Québec, Canada) has been developed by Fradet et $a l^{6}$ and was aimed at improving the low sensitivity of cytology. This fluorescence test combines three monoclonal antibodies. ${ }^{7}$ M344 and LDQ10, labelled 
with fluorescein, a green fluorescence, have been raised against mucin-like antigens. M344 is expressed by $71 \%$ of Ta-T1 tumors. ${ }^{8}$ 19A211, labelled with Texas red, recognizes a high molecular form of carcinoembryonic antigen (CEA) and is expressed by $90 \%$ of Ta-T1 tumors. ${ }^{8}$ Since the preliminary study by Mian et al, ${ }^{7}$ few additional studies have been published on the clinical usefulness of ImmunoCyt/uCyt ${ }^{\mathrm{TM}}$. This study was aimed at evaluating the clinical value of ImmunoCyt/uCyt ${ }^{\mathrm{TM}}$ in the diagnosis and follow-up of patients with urothelial carcinoma, and in cases with atypias suspicious for malignancy by cytology.

\section{Materials and methods}

\section{Population}

From May 1, 2000 to July 1, 2002, 1898 ImmunoCyt/ $\mathrm{uCyt}^{\mathrm{TM}}$ analyses have been consecutively performed on patients who presented for either urinary symptoms (dysuria, hematuria) or for follow-up of bladder tumor. This study includes 904 cases for which ImmunoCyt/uCyt ${ }^{\mathrm{TM}}$ has been performed along with cytology at the time of cystoscopy at the HôtelDieu de Québec University Hospital. Biopsies have been performed whenever a tumor was visible. Three categories of cystoscopy have been defined: negative, suspicious for cancer and positive. In this study, suspicious cases were included in the negative category. Urothelial tumor grades were defined according to the new World Health Organization/ International Society of Urological Pathology consensus classification of urothelial neoplasms ${ }^{9}$ and stages were classified according to the International Union against Cancer. ${ }^{10}$

\section{Specimen Collection}

Approximately $50-100 \mathrm{ml}$ of urine were collected. Part of the specimen was used for standard Papanicolaou stain. ${ }^{5}$ Three cytology categories were defined as negative, suspicious or positive for malignancy. In this study, cases with atypias suspicious for malignancy were included in the negative category.

\section{ImmunoCyt/uCyt ${ }^{\mathrm{TM}}$}

Approximately $20-40 \mathrm{ml}$ of urine were used for ImmunoCyt/uCyt ${ }^{\mathrm{TM}}$ assay. Samples were fixed with the same volume of $50 \%$ ethanol and $1 \mathrm{ml}$ of special fixative for $1 \mathrm{~h}$. Cells were collected by filtration through a membrane filter with a $50 \mathrm{ml}$ syringe. Cells were blotted on charged slides and fixed with a commercial cytology fixative or $70 \%$ isopropanol.

Staining was performed according to the company recommendations. Unstained slides were first incubated with 4 drops of a blocking solution for $15 \mathrm{~min}$ at room temperature in a humid chamber.
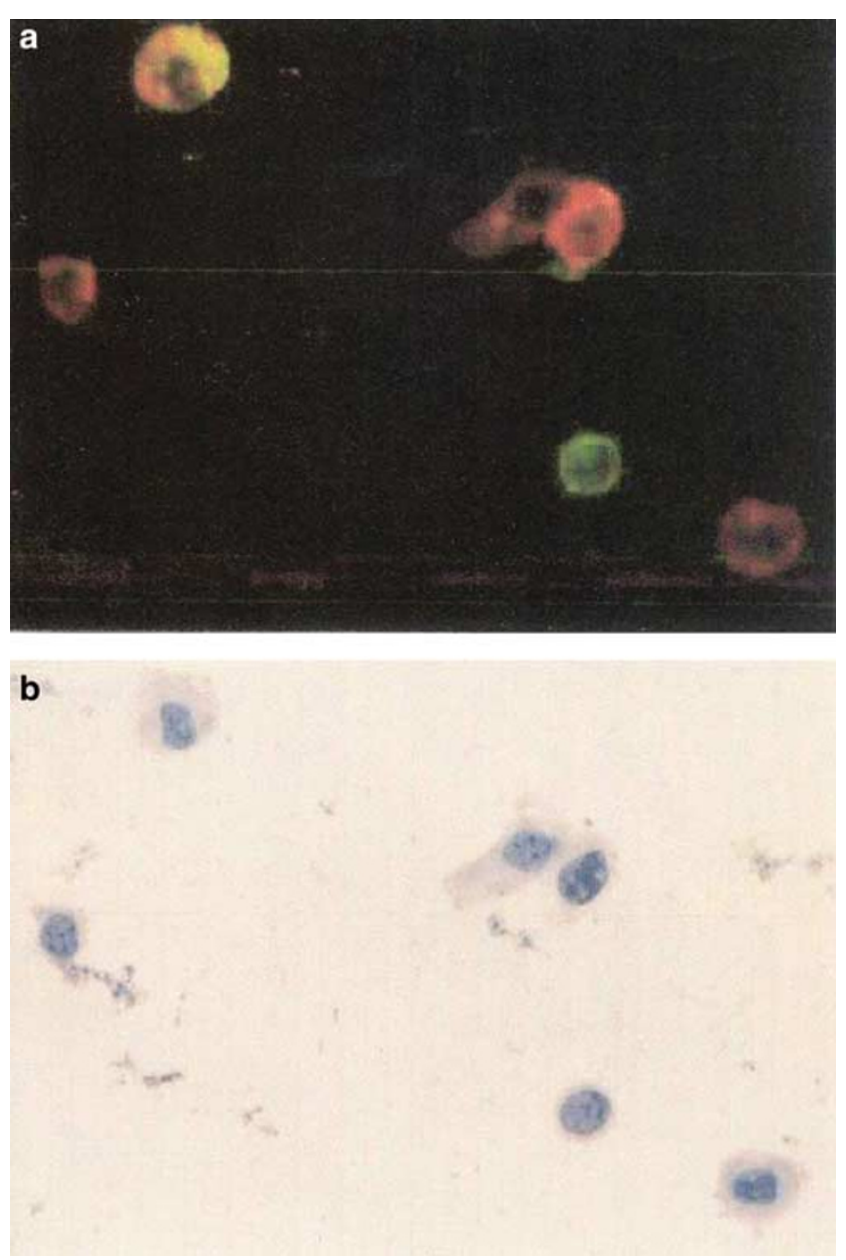

Figure 1 Presence of atypical urothelial cells as seen by (a) immunofluorescence with ImmunoCyt/uCyt ${ }^{\mathrm{TM}}(\times 400$, Texas red and fluorescein) and (b) light microsopy $(\times 400$, modified Papanicolaou stain) (reproduced with permission from Diagnocure Inc.).

The blocking solution was drained and slides were then incubated with the ImmunoCyt/uCyt ${ }^{\mathrm{TM}}$ cocktail for $1 \mathrm{~h}$ at room temperature in a dark room. Slides were then rinsed first in phosphate-buffered saline (PBS) containing $0.05 \%$ Tween and once in PBS. Slides were then conterstained in hematoxylin and mounted. Positive and negative control slides were prepared for each batch.

Slides were read using a fluorescence microscope with filters for fluorescein and Texas red detection. The number of cells positive for red and green fluorescence was calculated separately (Figure 1). Threshold positivity level was defined, as recommended by the company, as the presence of one green or one red fluorescent cell. Presence of tumor was confirmed by cystoscopy and biopsy.

\section{Data Analysis}

Sensitivity, specificity, as well as positive and negative predictive values of urine cytology and ImmunoCyt/uCyt ${ }^{\mathrm{TM}}$ were calculated using cysto- 
scopy and biopsy as reference. Differences in detection of bladder cancer between different categories of cytology or ImmunoCyt/uCyt ${ }^{\mathrm{TM}}$ was assessed using the $\chi^{2}$-test. Analyses were performed using one cell as threshold level.

\section{Results}

Of the 904 cases, 34 were excluded because they were inadequate for cytology or ImmunoCyt/uCyt ${ }^{\mathrm{TM}}$ either because of low cellularity or poor cell preservation. Of the 870 analyses included in this study, $734(84.4 \%)$ had no tumor present and 136 $(15.6 \%)$ had bladder cancer. Of those 136 analyses, tumor was histologically confirmed in 104 (77\%) cases and tumor was visualized by cystoscopy and fulgurated in $32(23 \%)$ cases. Of the 104 histologically proven, $31(30 \%)$ were of low malignant potential (LMP), 33 (32\%) were LG papillary carcinomas and 40 (39\%) were HG papillary carcinomas. Furthermore, 65 (63\%) were stage pTa, six $(6 \%)$ were pT1, $19(18 \%)$ were at least pT2 and $14(14 \%)$ were pTis. The age ranged from 21 to 95 years (average: $63.8 \%$ ).

\section{Cytology}

Of the 734 analyses with no tumor present, cytology was negative in 717 and, therefore, the specificity was high and reached $98 \%$ (Table 1). Of the 136 cases in which a tumor was present, cytology was positive in 39 , and the sensitivity of cytology was rather low at $29 \%$. Furthermore, the positive predictive value and negative predictive values were respectively $70 \%$ and $88 \%$ (Table 1 ). The sensitivity of cytology ranged from $7 \%$ in LMP tumors to $53 \%$ in HG carcinomas (Table 2). Sensitivity ranged from $12 \%$ in stage pTa to $50 \%$ in stage pTis and $67 \%$ in pT1 tumors (Table 2).

\section{ImmunoCyt/uCyt ${ }^{\mathrm{TM}}$}

Of the 136 cases for which a tumor was present, 100 were positive by ImmunoCyt/uCyt ${ }^{\mathrm{TM}}$ and the sensitivity was $74 \%$ (Table 1). Of the 734 cases without proven tumor, 453 were negative by ImmunoCyt/ $\mathrm{uCyt}^{\mathrm{TM}}$ and the specificity was $62 \%$. Furthermore, the positive predictive value and negative predictive value were, respectively, 26 and $93 \%$. The sensitivity and negative predictive value of ImmunoCyt/ $\mathrm{uCyt}^{\mathrm{TM}}$ were significantly higher than that of cytology whereas the specificity and positive predictive value were significantly lower $(P<0.05)$. The sensitivity of ImmunoCyt/uCyt ${ }^{\mathrm{TM}}$ ranged from $71 \%$ in LMP tumors to $85 \%$ in HG carcinomas (Table 2). Sensitivity ranged from $79 \%$ in stage pTa to $93 \%$ in stage pTis. The sensitivity of ImmunoCyt/uCyt ${ }^{\mathrm{TM}}$ was significantly higher than that of cytology for stages pTa and pTis and for all grades.

Table 1 Sensitivity, specificity, positive and negative predictive values of ImmunoCyt/uCyt ${ }^{\mathrm{TM}}$

\begin{tabular}{|c|c|c|c|c|c|c|c|c|c|c|}
\hline \multirow[t]{2}{*}{ Category } & \multicolumn{4}{|c|}{ Cytology } & \multicolumn{3}{|c|}{ ImmunoCyt/uCyt $t^{\mathrm{TM}}$} & \multicolumn{3}{|c|}{ Combined assays } \\
\hline & $\mathrm{n}$ & No. & $\%$ & $\mathrm{P}$ & $\mathrm{n}$ & No. & $\%$ & $\mathrm{n}$ & No. & $\%$ \\
\hline Sensitivity & 136 & 39 & 29 & $<0.001$ & 136 & 100 & 74 & 136 & 114 & 84 \\
\hline Specificity & 734 & 717 & 98 & $<0.001$ & 734 & 453 & 62 & 734 & 450 & 61 \\
\hline PPV & 56 & 39 & 70 & $<0.001$ & 382 & 100 & 26 & 398 & 114 & 29 \\
\hline NPV & 814 & 717 & 88 & 0.005 & 488 & 453 & 93 & 472 & 450 & 95 \\
\hline
\end{tabular}

PPV: positive predictive value; NPV: negative predictive value; $n$ : total number analyses (reference number); No.: number of positive or a negative results for the test; $P$ : McNemar $P$-value for comparison of Cytology vs ImmunoCyt $/ \mathrm{uCyt}^{\mathrm{TM}}$.

Table 2 Sensitivity of ImmunoCyt/uCyt ${ }^{\mathrm{TM}}$ according to stage and grade of urothelial cancer

\begin{tabular}{|c|c|c|c|c|c|c|c|c|}
\hline \multirow[t]{2}{*}{ Category } & \multicolumn{4}{|c|}{ Cytology } & \multicolumn{2}{|c|}{ ImmunoCyt/uCyt } & \multicolumn{2}{|c|}{ Combined assays } \\
\hline & $\mathrm{n}$ & No. & $\%$ & $\mathrm{P}$ & No. & $\%$ & No. & $\%$ \\
\hline \multicolumn{9}{|l|}{ Stage } \\
\hline pTa & 65 & 8 & 12 & $<0.001$ & 51 & 79 & 51 & 79 \\
\hline pT1 & 6 & 4 & 67 & 0.32 & 5 & 83 & 5 & 83 \\
\hline$\geq \mathrm{pT} 2$ & 19 & 9 & 47 & 0.16 & 13 & 68 & 15 & 79 \\
\hline CIS & 14 & 7 & 50 & 0.034 & 13 & 93 & 14 & 100 \\
\hline \multicolumn{9}{|l|}{ Histology } \\
\hline LMP & 31 & 2 & 7 & $<0.001$ & 22 & 71 & 22 & 71 \\
\hline LG & 33 & 6 & 18 & $<0.001$ & 26 & 79 & 26 & 79 \\
\hline HG & 40 & 21 & 53 & 0.003 & 34 & 85 & 37 & 93 \\
\hline
\end{tabular}

$n$ : Total number analyses (reference number); No.: number of positive results for the test; CIS: carcinoma in situ; LMP: papillary neoplasm of low malignant potential; LG: low-grade papillary carcinoma; HG: high-grade papillary carcinoma; $P$ : McNemar $P$-value for comparison of Cytology vs ImmunoCyt/uCyt ${ }^{\mathrm{TM}}$. 


\section{Combination of Cytology and ImmunoCyt/uCyt ${ }^{\mathrm{TM}}$}

Overall sensitivity was improved by combining cytology with ImmunoCyt/uCyt ${ }^{\mathrm{TM}}$. Of the 136 cases for which a tumor was present, 114 were positive by cytology and ImmunoCyt/uCyt ${ }^{\mathrm{TM}}$ and the sensitivity was $84 \%$ compared to $74 \%$ with ImmunoCyt/uCyt ${ }^{\mathrm{TM}}$ alone (Table 1). The sensitivity of combined tests ranged from $71 \%$ in LMP tumors to $93 \%$ in HG carcinomas and from $79 \%$ in stage pTa to $100 \%$ in stage pTis (Table 2). It was fairly comparable to ImmunoCyt/uCyt ${ }^{\mathrm{TM}}$ alone.

\section{Follow-up of Patients with Negative Cystoscopy and Positive Cytology and ImmunoCyt/uCyt ${ }^{\mathrm{TM}}$}

Table 3 shows the recurrence rate of patients whose cystoscopy was negative when the test was performed. It shows that, with a negative cytology, a tumor appeared in 6\% of cases, 2-6 months after the negative cystoscopy, while a tumor was present in $7 \%$ of cases with a negative ImmunoCyt/uCyt ${ }^{\mathrm{TM}}$. However, of the 10 cases with a positive cytology, five $(50 \%)$ developed a tumor in this time range $(P<0.0001)$. Of the 104 cases with a positive ImmunoCyt/uCyt ${ }^{\mathrm{TM}}, 19(18 \%)$ developed a tumor, 2-6 months later $(P=0.0057)$. Interestingly, after 6 months, $6-11 \%$ of patients developed a recurrence, whether cytology and/or ImmunoCyt/uCyt ${ }^{\mathrm{TM}}$ was positive or negative (Table 3 ).

\section{Suspicious Cases by Cytology}

Table 4 shows the results of ImmunoCyt/uCyt ${ }^{\mathrm{TM}}$ in cases that were suspicious for malignancy by cytology. A total of 109 analyses were interpreted as suspicious. Of them, a tumor was present in 30

Table 3 Value of cytology and ImmunoCyt/uCyt ${ }^{\mathrm{TM}}$ to predict tumor recurrence in patients with a negative cystoscopy and a minimum of 2 months of follow-up

\begin{tabular}{|c|c|c|c|c|c|}
\hline \multirow[t]{3}{*}{ Test } & \multirow[t]{3}{*}{$\mathrm{n}$} & \multicolumn{4}{|c|}{ Recurrence } \\
\hline & & \multicolumn{2}{|c|}{ 2-6 months FU } & \multicolumn{2}{|c|}{$>6$ months $F U$} \\
\hline & & $\mathrm{n}$ & $\%$ & $\mathrm{n}$ & $\%$ \\
\hline \multicolumn{6}{|l|}{ Cytology } \\
\hline Negative & 187 & 12 & 6 & 19 & 10 \\
\hline Suspicious & 42 & 11 & 26 & 3 & 7 \\
\hline Positive & 10 & 5 & 50 & 1 & 10 \\
\hline$P$-value & & \multicolumn{2}{|c|}{$<0.0001$} & \multicolumn{2}{|c|}{0.8349} \\
\hline \multicolumn{6}{|c|}{ ImmunoCyt/uCyt ${ }^{\mathrm{TM}}$} \\
\hline Negative & 135 & 9 & 7 & 13 & 10 \\
\hline Positive & 104 & 19 & 18 & 10 & 10 \\
\hline$P$-value & & \multicolumn{2}{|c|}{0.0057} & \multicolumn{2}{|c|}{0.9970} \\
\hline
\end{tabular}

n: Number of analyses; FU: follow-up.
Table 4 Analyses with suspicious cytology

\begin{tabular}{|c|c|c|c|c|c|c|}
\hline \multirow[t]{3}{*}{ ImmunoCyt/uCyt $^{\mathrm{TM}}$} & \multicolumn{2}{|c|}{$\begin{array}{c}\text { Total } \\
\text { suspicious } \\
\text { (109 cases) }\end{array}$} & \multicolumn{4}{|c|}{ Tumor } \\
\hline & \multirow[t]{2}{*}{$\mathrm{n}$} & \multirow[t]{2}{*}{$\%$} & \multicolumn{2}{|c|}{$\begin{array}{c}\text { Absent } \\
\text { (79 cases) }\end{array}$} & \multicolumn{2}{|c|}{$\begin{array}{c}\text { Present } \\
(30 \text { cases) }\end{array}$} \\
\hline & & & $\mathrm{n}$ & $\%$ & $\mathrm{n}$ & $\%$ \\
\hline Negative & 35 & 32 & 27 & 34 & 8 & 27 \\
\hline Positive & 74 & 68 & 52 & 66 & 22 & 73 \\
\hline$P$-value & & & \multicolumn{4}{|c|}{0.362} \\
\hline
\end{tabular}

$n$ : Number of analyses.

cases. Of the 24 cases with a histologically confirmed tumor, six $(25 \%)$ were LMP tumors, nine $(38 \%)$ were LG carcinomas and nine $(38 \%)$ were HG carcinomas. Furthermore, $16(67 \%)$ were pTa, one $(4 \%)$ was pT1, four $(17 \%)$ were pT2 or more and three $(13 \%)$ were pTis. Of the 109 cases, ImmunoCyt/uCyt ${ }^{\mathrm{TM}}$ was positive in $74(68 \%)$. Of the 30 cases with a tumor present, ImmunoCyt/uCyt ${ }^{\mathrm{TM}}$ was positive in $22(73 \%)$. Of the 79 cases without tumor present, $66 \%$ had a positive ImmunoCyt/uCyt ${ }^{\mathrm{TM}}$. The difference in ImmunoCyt/uCyt ${ }^{\mathrm{TM}}$ expression between cases with or without tumor in the bladder was not significant $(P=0.362)$.

\section{Discussion}

With 870 analyses, our study on ImmunoCyt/uCyt ${ }^{\mathrm{TM}}$ is the largest published to date. Most prior studies confirm that ImmunoCyt/uCyt ${ }^{\mathrm{TM}}$ significantly improves the sensitivity of cytology. With such high sensitivity and negative predictive value, urologists have access to a urine test that may help to reduce the frequency of follow-up cystoscopies. Indeed, in most studies, the sensitivity of ImmunoCyt/uCyt ${ }^{\mathrm{TM}}$ combined with cytology exceeds $80 \%$ when a threshold of 1 red or 1 green fluorescent cell is used. ${ }^{6,7,11-14}$ Our sensitivity of $84 \%$ means that, if a tumor is present, it would most likely be detected by the test. Therefore, if the test is positive, a cystoscopy should be performed and, if no tumor is seen, a tumor from the upper urinary tract should be suspected and ruled out. Our negative predictive value of $95 \%$ is comparable to other studies which ranged from $81^{15}$ to $97 \% .^{7}$ Such a high-negative predictive value means that, if the test is negative, patients undergoing a standard cystoscopy protocol for a history of bladder cancer are unlikely to have recurrent bladder tumor and that the next control cystoscopy may be postponed. In young patients investigated for urinary symptoms, presence of a bladder tumor is unlikely with a negative test and cystoscopy might be omitted. In our study, the 
positive predictive value of ImmunoCyt/uCyt ${ }^{\mathrm{TM}}$ was much lower $(26 \%)$ than that of cytology $(70 \%)$. This might suggest that such false positivity would lead to unnecessary cystoscopies. However, since current follow-up protocols for the management of superficial bladder cancer imply systematic cystoscopies at fixed intervals, more false-positive cases will not result in more frequent cystoscopies.

A number of noninvasive tests to detect urinary bladder cancer have been developed. Overall sensitivities and specificities are summarized in Table 5. BTA Stat and BTA TRAK (Bard Diagnostics, Redmond, WA, USA) detect proteins (complement factor H-related protein) present in urine and can be used at a physician's office. ${ }^{4}$ However, although these tests may improve the sensitivity of cytology to as high as $78 \%$, such improvement remains modest for LG, low-stage tumors for which it merely reaches $50 \%{ }^{4}$ Furthermore, the overall specificity is relatively low, partly because those proteins may be increased in urine of patients with non neoplastic diseases such as inflammatory conditions. ${ }^{3}$ Furthermore, certain tests must be performed in specialized laboratories. For example, NMP22 (Matritech, Cambridge, MA) detects a nuclear mitotic apparatus protein released in urine but must be performed in a reference laboratory only. ${ }^{4}$ Encouraging high sensitivities have been reported with telomerase ${ }^{4}$ and microsatellite analyses, ${ }^{16}$ but these techniques require PCR amplification, are labor-intensive and need to be performed by trained technicians. Vysis uroVision (Vysis Inc., Downers Grove, IL, USA) provides good sensitivity and specificity (81 and $96 \%$ respectively $)^{17}$ but, as for any fluorescence in situ hybridization (FISH) technology, this test is better performed in a reference laboratory and the interpretation is more time-consuming than immunofluorescence. ImmunoCyt/uCyt ${ }^{\mathrm{TM}}$ has been developed as a complement to cytology and can be performed by the same personnel trained for cytology screening. Furthermore, although this test cannot be performed in a physician's office, good sensitivities and specificities were obtained even in smaller laboratories, ${ }^{11}$ provided that quality control programs are well established. Thus, ImmunoCyt/

Table 5 Current data on sensitivity and specificity of commercially available and experimental urine tests

\begin{tabular}{ccc}
\hline Test & Sensitivity & Specificity \\
Low-high (Median) & Lowhigh \\
(Median)
\end{tabular}

Cytology $21-28$

BTA stat ${ }^{21,23-25,28-31}$

BTA trak ${ }^{21,26,30-32}$

NMP22 $21,22,27,28,31,33$

Telomerase ${ }^{4,17,22}$

Microsatellites ${ }^{16}$

FISH $^{17}$

ImmunoCyt/

$\mathrm{uCyt}^{\mathrm{TM} 6,7,11,13-15,18,20,21}$
$\mathrm{uCyt}^{\mathrm{TM}}$ does not necessarily need to be centralized in large central laboratories.

The high 'false-positive' rate of ImmunoCyt/uCyt ${ }^{\mathrm{TM}}$ may lead the urologist to conclude that the patient has no urothelial tumor in cases of a positive test with a negative cystoscopy. However, $18 \%$ of patients with 'false-positive' ImmunoCyt/uCyt ${ }^{\mathrm{TM}}$ developed a recurrence, 2-6 months after the negative cystoscopy, compared $7 \%$ only in those with negative ImmunoCyt/uCyt $^{\mathrm{TM}}(P=0.006)$. Our study shows also that $50 \%$ of patients with 'false-positive' cytologies developed a recurrence, 2-6 months following a negative cystoscopy, compared to only $6 \%$ of recurrences in those with a negative cytology $(P<0.0001)$. Even though cytology had an apparent higher predictive rate than ImmunoCyt/uCyt ${ }^{\mathrm{TM}}$ (50 vs 18\%), ImmunoCyt/uCyt ${ }^{\mathrm{TM}}$ predicted the occurrence of 19 tumors, as opposed to only five by cytology, because of the low sensitivity of the latter. Interestingly, while positive ImmunoCyt/uCyt ${ }^{\mathrm{TM}}$ was not predictive of increased recurrences compared to negative ImmunoCyt/uCyt ${ }^{\mathrm{TM}}$, after 6 months of follow-up, Piaton et $a 1^{14}$ found that the test strongly predicted recurrences at 1 year (47.0 vs $11.9 \%$ for patients with positive and negative tests, respectively).

Urine cytology is the standard noninvasive method to detect cancer cells in urine. It has been used for decades and its advantages and limitations are well known. Cytology diagnosis is based, however, on subjective criteria that account for difficulties in interpretation. ${ }^{5}$ Among them is the difficulty to recognize LG tumor cells from normal cells, even when papillary structures are present. This explains the low sensitivity of cytology for LG tumors in the literature and in our hands. It is estimated that only $30-60 \%$ of papillomas and LMP tumors will exfoliate with sufficient cytologic abnormalities to be recognized as neoplastic. ${ }^{5}$ Because of their low density in urine and their mild atypias, LG tumors require considerable screening time from both cytologists and pathologists and the overall accuracy of the test is poor. The high sensitivity of ImmunoCyt/uCyt ${ }^{\mathrm{TM}}$ is helpful with those tumors because abnormal fluorescent cells are readily visible even if few events are present and their detection does not rely on subtle subjective morphologic criteria. Therefore, the high sensitivity of ImmunoCyt/uCyt ${ }^{\mathrm{TM}}$ may help pathologists and cytologists to save time at screening slides of LG tumors.

ImmunoCyt/uCyt ${ }^{\mathrm{TM}}$ may also improve the particularly low sensitivity of cytology at detecting upper urinary tract urothelial cell carcinoma. Indeed, in one study, while no LMP tumors and $17 \%$ of LG carcinomas only were detected by cytologic examination in upper urinary tract, the sensitivity raised to $33 \%$ of LMP tumors and $100 \%$ of LG carcinomas with ImmunoCyt/uCyt ${ }^{\mathrm{TM}} \cdot{ }^{18}$

Our study concurs with several others and confirms marked improvement of sensitivity by incorporating ImmunoCyt/uCyt ${ }^{\mathrm{TM}}$ with cytology. Most studies reported results comparable or better 
than ours with two exceptions. ${ }^{15,19}$ By contrast, results were found fairly reproducible in a recently reported ten-Center French study. ${ }^{20}$ However, it is clear that the validity of the test relies largely on the quality of the training of cytologists and on the presence of well-established quality control programs in cytology laboratories. The combination of ImmunoCyt/uCyt ${ }^{\mathrm{TM}}$ with cytology may also help to detect nonvisible HG cancers, which are often missed by the cystoscopist. In our study, as in others, ${ }^{7,14,20}$ all carcinomas in situ were found by combining cytology and ImmunoCyt/uCyt ${ }^{\mathrm{TM}}$, while $50 \%$ had either negative or suspicious cytology.

Reactive urothelial cells show mild abnormalities that are shared by LG neoplastic cells and require considerable screening time from pathologists and cytologists. These include a mildly increased nuclear:cytoplasmic ratio and the presence of papillary aggregates without significant nuclear atypias. These features are helpful to detect LG cancer cells in urine but may also occur with lithiasis or other inflammatory process or in patients submitted to cytotoxic drugs or radiation therapy. ${ }^{5}$ The presence of significant abnormalities warrants a diagnosis of atypias suspicious for malignancy. In our study, $28 \%$ of suspicious cases had a tumor present. Those tumors were not insignificant since $41 \%$ were HG carcinomas. However, of the 30 cases with tumor and a suspicious cytology, $73 \%$ had a positive ImmunoCyt/uCyt ${ }^{\mathrm{TM}}$. The availability of a complement to cytology for such cases may help to save professional time since suspicious cells usually require considerable work from both the cytologist who first screens the slides and the pathologist who has to make a final diagnosis.

We conclude that ImmunoCyt/uCyt ${ }^{\mathrm{TM}}$ is a useful adjunct to cytology to detect both LG and HG tumors, including carcinoma in situ in urine specimens. ImmunoCyt/uCyt ${ }^{\mathrm{TM}}$ may also be used to help cytologists and pathologists save screening time, more specifically in cases with atypias suspicious for malignancy and in low-stage and LG tumors. Finally, a positive ImmunoCyt/uCyt ${ }^{\mathrm{TM}}$ with a negative cystoscopy predicted higher recurrence rate in the next few months.

\section{Acknowledgements}

Dr Fradet is also Vice-President of DiagnoCure Inc., which produces the ImmunoCyt/uCyt $+{ }^{\mathrm{TM}}$ test. There is no duality of interest for other authors. We are thankful to Louise Pelletier, Marlène Castilloux, Rachel Frigon, Louise Paré, Louise Lafleur, Hélène Martin and Claire Lagacé, cytotechnologists at the Centre Hospitalier Universitaire de Québec, for their expert participation to the reading of ImmunoCyt/uCyt ${ }^{\mathrm{TM}}$. We are also thankful to Doctors Claude Delage and Chantal Veilleux, Hôtel-Dieu de Québec, for their collaboration in the setting of the test in our hospital.

\section{References}

1 Koch M, Hill GB, McPhee MS. Factors affecting recurrence rates in superficial bladder cancer. J Natl Cancer Inst 1986;76:1025-1029.

2 Malmstrom PU, Busch C, Norlen BJ. Recurrence, progression and survival in bladder cancer. A retrospective analysis of 232 patients with greater than or equal to 5-year follow-up. Scand J Urol Nephrol 1987;21:185-195.

3 Stein JP, Grossfeld GD, Ginsberg DA, et al. Prognostic markers in bladder cancer: a contemporary review of the literature. J Urol 1998;160:645-659.

4 Lokeshwar VB, Soloway MS. Current bladder tumor tests: does their projected utility fulfill clinical necessity? J Urol 2001;165:1067-1077.

5 Murphy WM. Current status of urinary cytology in the evaluation of bladder neoplasms. Hum Pathol 1990;21:886-896.

6 Fradet Y, Lockhart C, the Immunocyt trialists. Performance characteristics of a new monoclonal antibody test for bladder cancer: Immunocyt ${ }^{\mathrm{TM}}$. Can J Urol 1997;4:400-405.

7 Mian C, Pycha A, Wiener H, et al. Immunocyt: a new tool for detecting transitional cell cancer of the urinary tract. J Urol 1999;161:1486-1489.

8 Allard P, Fradet Y, Tetu B, et al. Tumor-associated antigens as prognostic factors for recurrence in 382 patients with primary transitional cell carcinoma of the bladder. Clin Cancer Res 1995;1:1195-1202.

9 Epstein JI, Amin MB, Reuter VR, et al. The World Health Organization/International Society of Urological Pathology consensus classification of urothelial (transitional cell) neoplasms of the urinary bladder. Bladder Consensus Conference Committee. Am J Surg Pathol 1998;22:1435-1448.

10 International Union Against Cancer. TNM Classification of Malignant Tumours, 4th edn. Springer-Verlag: Geneva, 1987.

11 Olsson H, Zackrisson B. ImmunoCyt a useful method in the follow-up protocol for patients with urinary bladder carcinoma. Scand J Urol Nephrol 2001;35: 280-282.

12 Pérez Barrios A, Alberti Masgrau N, De Agustin P. The value of immunocyt ${ }^{\mathrm{TM}}$ in the diagnosis of bladder carcinoma. Urol Integr Invest 2001;6:109-111.

13 Lodde M, Mian C, Negri G, et al. Role of uCyt+ in the detection and surveillance of urothelial carcinoma. Urology 2003;61:243-247.

14 Piaton E, Daniel L, Verriele V, et al. Improved detection of urothelial carcinomas with fluorescence immunocytochemistry (uCyt+ assay) and urinary cytology: results of a French Prospective Multicenter Study. Lab Invest 2003;83:845-852.

15 Vriesema JL, Atsma F, Kiemeney LA, et al. Diagnostic efficacy of the Immunocyt test to detect superficial bladder cancer recurrence. Urology 2001;58:367-371.

16 van Rhijn BW, Lurkin I, Kirkels WJ, et al. Microsatellite analysis-DNA test in urine competes with cystoscopy in follow-up of superficial bladder carcinoma: a phase II trial. Cancer 2001;92:768-775.

17 Halling KC, King W, Sokolova IA, et al. A comparison of BTA stat, hemoglobin dipstick, telomerase and Vysis UroVysion assays for the detection of urothelial carcinoma in urine. J Urol 2002;167:2001-2006.

18 Lodde M, Mian C, Wiener $\mathrm{H}$, et al. Detection of upper urinary tract transitional cell carcinoma with 
ImmunoCyt: a preliminary report. Urology 2001;58: 362-366.

19 Feil G, Zumbragel A, Paulgen-Nelde HJ, et al. Accuracy of the ImmunoCyt assay in the diagnosis of transitional cell carcinoma of the urinary bladder. Anticancer Res 2003;23:963-967.

20 Pfister C, Chautard D, Devonec M, et al. Immunocyt test improves the diagnostic accuracy of urinary cytology: results of a French multicenter study. J Urol 2003;169:921-924.

21 Lodde M, Mian C, Negri G, et al. Effect of intravesical instillation on performance of uCYT+ test. Urology 2004;63:878-881.

22 Ramakumar S, Bhuiyan J, Besse JA, et al. Comparison of screening methods in the detection of bladder cancer. J Urol 1999;161:388-394.

23 Sarosdy MF, Hudson MA, Ellis WJ, et al. Improved detection of recurrent bladder cancer using the Bard BTA stat Test. Urology 1997;50:349-353.

24 Gutierrez Banos JL, Henar Rebollo RM, Antolin Juarez FM, et al. Usefulness of the BTA STAT Test for the diagnosis of bladder cancer. Urology 2001;57:685-689.

25 Leyh H, Hall R, Mazeman E, et al. Comparison of the Bard BTA test with voided urine and bladder wash cytology in the diagnosis and management of cancer of the bladder. Urology 1997;50:49-53.

26 Chautard D, Daver A, Bocquillon V, et al. Comparison of the Bard Trak test with voided urine cytology in the diagnosis and follow-up of bladder tumors. Eur Urol 2000;38:686-690.
27 Lahme S, Bichler KH, Feil G, et al. Comparison of cytology and nuclear matrix protein 22 for the detection and follow-up of bladder cancer. Urol Int 2001;66:72-77.

28 Giannopoulos A, Manousakas T, Mitropoulos D, et al. Comparative evaluation of the BTAstat test, NMP22, and voided urine cytology in the detection of primary and recurrent bladder tumors. Urology 2000;55: 871-875.

29 Mian C, Lodde M, Haitel A, et al. Comparison of two qualitative assays, the UBC rapid test and the BTA stat test, in the diagnosis of urothelial cell carcinoma of the bladder. Urology 2000;56:228-231.

30 Irani J, Desgrandchamps F, Millet C, et al. BTA stat and BTA TRAK: a comparative evaluation of urine testing for the diagnosis of transitional cell carcinoma of the bladder. Eur Urol 1999;35:89-92.

31 Serretta V, Pomara G, Rizzo I, et al. Urinary BTA-stat, BTA-trak and NMP22 in surveillance after TUR of recurrent superficial transitional cell carcinoma of the bladder. Eur Urol 2000;38:419-425.

32 Ellis WJ, Blumenstein BA, Ishak LM, et al. Clinical evaluation of the BTA TRAK assay and comparison to voided urine cytology and the Bard BTA test in patients with recurrent bladder tumors. The Multi Center Study Group. Urology 1997;50:882-887.

33 Stampfer DS, Carpinito GA, Rodriguez-Villanueva J, et al. Evaluation of NMP22 in the detection of transitional cell carcinoma of the bladder. J Urol 1998; 159:394-398. 